\title{
Whakawhanaungatanga: the importance of
} culturally meaningful connections to improve uptake of pulmonary rehabilitation by Māori with COPD - a qualitative study

\author{
This article was published in the following Dove Press journal: \\ International Journal of COPD \\ 9 March 2016 \\ Number of times this article has been viewed
}

\section{William MM Levack \\ Bernadette Jones \\ Rebecca Grainger \\ Pauline Boland \\ Melanie Brown \\ Tristram R Ingham}

Department of Medicine, University of Otago, Wellington, New Zealand
Correspondence: William MM Levack Department of Medicine, University of Otago, Wellington, PO Box 7343, Wellington 6242, New Zealand

Tel +6449186279

Fax +64 43895427
Email william.levack@otago.ac.nz

Background: Pulmonary rehabilitation is known to improve function and quality of life for people with chronic obstructive pulmonary disease (COPD). However, little research has been conducted on the influence of culture on experiences of pulmonary rehabilitation. This study examined factors influencing uptake of pulmonary rehabilitation by Māori with COPD in New Zealand.

Method: Grounded theory nested within kaupapa Māori methodology. Transcripts were analyzed from interviews and focus groups with 15 Māori and ten New Zealand non-Māori invited to attend pulmonary rehabilitation for COPD. Māori participants had either attended a mainstream hospital-based program, a community-based program designed "by Māori, for Māori”, or had experienced both.

Results: Several factors influencing uptake of pulmonary rehabilitation were common to all participants regardless of ethnicity: 1) participants' past experiences (eg, of exercise; of health care systems), 2) attitudes and expectations, 3) access issues (eg, time, transport, and conflicting responsibilities), and 4) initial program experiences. These factors were moderated by the involvement of family and peers, interactions with health professionals, the way information on programs was presented, and by new illness events. For Māori, however, several additional factors were also identified relating to cultural experiences of pulmonary rehabilitation. In particular, Māori participants placed high value on whakawhanaungatanga: the making of culturally meaningful connections with others. Culturally appropriate communication and relationship building was deemed so important by some Māori participants that when it was absent, they felt strongly discouraged to attend pulmonary rehabilitation. Only the more holistic services offered a program in which they felt culturally safe and to which they were willing to return for ongoing rehabilitation.

Conclusion: Lack of attention to cultural factors in the delivery of pulmonary rehabilitation may be a barrier to its uptake by indigenous, minority ethnic groups, such as New Zealand Māori. Indigenous-led or culturally responsive health care interventions for COPD may provide a solution to this issue.

Keywords: COPD, pulmonary rehabilitation, health care access, cultural safety, indigenous peoples, qualitative research

\section{Introduction}

It is now well established that indigenous peoples in colonized countries, an estimated 370 million people worldwide, ${ }^{1}$ endure significantly poorer health than their nonindigenous counterparts. ${ }^{2}$ Compared to others in their native lands, indigenous peoples suffer 
higher rates of cancer, cardiovascular disease, respiratory disease, stroke, and diabetes and have lower life expectancy and higher infant mortality. ${ }^{3-6}$ These findings hold true for indigenous peoples regardless of whether they live in wealthy countries, such as Australia, New Zealand, Canada, or USA, or in developing nations. ${ }^{3-6}$

This paper explores the interactions between ethnicity and uptake of health care services in the context of pulmonary rehabilitation for chronic obstructive pulmonary disease (COPD) in New Zealand. Pulmonary rehabilitation provides an excellent microcosm within which to examine cultural issues in health care for a number of reasons. First, COPD is a significant health problem. In New Zealand, it affects $14 \%$ of adults over 40 years of age $^{7}$ and is the fourth leading cause of death. ${ }^{8}$ Māori, the indigenous people of New Zealand, experience significant inequities in COPD including higher prevalence, hospitalizations, and deaths associated with the condition compared with non-Māori. ${ }^{9}$ Second, pulmonary rehabilitation is a largely standardized intervention, usually involving 6-12 weeks of exercise and self-management education, with little variation in its implementation either within New Zealand ${ }^{10}$ or internationally. ${ }^{11}$ Third, pulmonary rehabilitation is an important intervention of known effectiveness, offering one of the few successful strategies to enhance physical function and quality of life ${ }^{12}$ and reducing hospital admissions and deaths among people with COPD. ${ }^{13}$

Finally, access to pulmonary rehabilitation is known to be a problem for all people with COPD, internationally. ${ }^{11}$ Known barriers to greater uptake of pulmonary rehabilitation include problems with transport and timing of programs, disruption of usual routines, and the distance required to travel to program venues. ${ }^{14}$ However, although it has been recognized that "in practice, the details of program construction and setting will vary with different cultures and health care systems", ${ }^{15}$ very little research has been conducted on cultural factors in the delivery of pulmonary rehabilitation. Of the eleven studies identified in a systematic review examining factors influencing uptake and completion of pulmonary rehabilitation, ${ }^{14}$ ethnicity was either not reported or reported in insufficient numbers to allow meaningful analysis.

Reasons for poorer health among indigenous peoples are complex. In New Zealand for instance, socioeconomic status alone is insufficient to explain major health inequities. In 2000, Māori in the top socioeconomic brackets of New Zealand society were found to be experiencing significantly lower life expectancy than even the most socioeconomically deprived non-Māori. ${ }^{16}$ Despite health gains in recent decades, the average life expectancy for Māori today is still 7.1 years shorter than non-Māori. ${ }^{17}$ Internationally, poorer health among indigenous peoples has not only been associated with poverty, lower levels of education, and unemployment but also with the ongoing effects of colonization, loss of connections with land and cultural identity, ${ }^{3,18}$ and with experiences of internalized, interpersonal and institutional racism. ${ }^{19-22}$ Timely and reliable access to health care services is also a significant problem for indigenous peoples worldwide..$^{23-28}$

In this paper, we draw on the concept of cultural safety in order to understand the experiences of Māori with COPD, accessing pulmonary rehabilitation in New Zealand. Cultural safety was first developed by Māori nurse leaders in response to dissatisfaction with the way culture had been previously conceptualized and addressed in mainstream health care in New Zealand. ${ }^{29}$ Cultural safety is characterized by a focus on the experiences of the recipients of health care services, with unsafe cultural practice being defined as "any action which diminishes, demeans or disempowers the cultural identity and well being of an individual" (p. 28). ${ }^{30}$ Cultural safety centers on identifying, analyzing, and responding to power relations within health care interactions. At a practical level, this relates to how services are organized, offered, and delivered; how recipients of services are involved in decisions regarding their health care; and the manner in which health professionals interact with their patients or clients. ${ }^{30}$ For instance, cultural safety practice includes creating a physical and social space in which recipients of health care feel safe and well cared for. As cultural safety arises in part from the beliefs, attitudes, and values of those who deliver health services, it also requires health care providers to acknowledge historical and current causes of health inequities, and to recognize and address the impact of the health professionals' own history, culture, and beliefs on relationships they have with the people who receive their services. ${ }^{31}$ Cultural safety has been contrasted with transcultural care ${ }^{32}$ and notions of cultural sensitivity, where ethnic minorities are treated as exotic: to be observed, learned about, and accommodated for in clinical practice. ${ }^{33}$ Such practice, the proponents of cultural safety argue, can risk reducing culture in clinical practice to a collection of checklists, reinforcing stereotypes and failing to address the very power imbalances that can make clinical services unsafe for indigenous peoples and other minority groups. ${ }^{29,34}$ The relevance of these ideas to the uptake and completion of pulmonary rehabilitation by Māori in New Zealand is explored in this paper. 


\section{Methods}

\section{Design}

This study employed grounded theory methods,${ }^{35}$ nested within principles of kaupapa Māori methodology. Kaupapa Māori methodology is a Māori way of doing research to serve Māori needs and objectives. ${ }^{36-38}$ Developed in part in reaction to dominant westernized positivistic research, the main principle that has been ascribed to kaupapa Māori research is that of tino rangatiratanga (ie, self-determination, sovereignty, governance, autonomy, and independence).$^{39}$ Kaupapa Māori research requires full recognition of Māori cultural values and systems, observance of Māori protocols and customs during research processes, and requires Māori control over the assumptions, values, and priorities of the research, and also over the interpretation and use of research findings. ${ }^{36,40}$ In the case of our research, the work was undertaken collaboratively by both Māori (BJ and TI) and non-Māori (WL, RG, PB, and MB) researchers. Māori researchers controlled and directed both the data collection and analysis processes in collaboration with non-Māori researchers. This ensured that the portrayal of Māori experiences was grounded in a cultural reality. ${ }^{38}$

Grounded theory was used in this study because it is a research method suitable for uncovering new perspectives on people's beliefs and lived experiences. An abbreviated version of grounded theory rather than full grounded theory was used in this study. Abbreviated grounded theory is an approach in which study data are collected and analyzed following:

The principles of grounded theory (ie, the processes of coding and constant comparative analysis); [but where] theoretical sensitivity, theoretical saturation, and negative cases analysis can only be implemented within the texts that are being analysed. ${ }^{41}$

We used a constructivist epistemology in our application of grounded theory. ${ }^{35}$ Constructivism is a belief that knowledge and meaning does not reside in objects in the world but is created through the engagement of meaning-makers (ie, people) with the world. ${ }^{42}$ This approach to grounded theory fits well with a kaupapa Māori methodology as it permits cultural understandings of the world in the production, analysis, and interpretation of research data.

\section{Ethics and cultural consultation}

Prior to the start of this project, consultation was undertaken with a Research Advisory Group for Māori at a local hospital that represents mana whenua (tribal authority and jurisdiction over land or territory) in our region, with Māori nurses who ran COPD support services locally, and with Te Ruanga o Ngāi Tahu - the tribal group with whom our University has an enduring collaborative relationship. Community consultation was also undertaken at a local marae (a Māori community facility that serves spiritual and social functions) with Māori staff from the Tu Kotahi Māori Asthma Trust, who were involved with delivering Māori respiratory and pulmonary rehabilitation services. We sought to gain their input into the design, methods, and research processes prior to the commencement of this project. ${ }^{43}$ Ethical approval for this study was provided by the Central Health and Disability Ethics Committee (CEN/11/EXP/062).

\section{Participant recruitment}

To be included in the study, participants were required to be adults (above 18 years of age) with COPD who had been offered a place on a pulmonary rehabilitation program within the past 6 months. Our study focused on the experiences of people in two types of pulmonary rehabilitation programs: 1) mainstream, hospital-based programs, which were westernized and led by non-Māori health practitioners, and 2) a marae-based program that was run by Māori, for Māori. The marae-based program was run by a local Māori health care provider group, which had been offering respiratory nurse services since 1995 . For this study we recruited Māori people with COPD, but, for purposes of comparison, we also aimed to recruit non-Māori people with COPD. The Māori participants included people who had only experienced one type of pulmonary rehabilitation (either hospital-based or marae-based) and people who had experienced both. The nonMāori participants had only experienced the hospital-based programs. We also endeavored to recruit people with COPD who had been offered a place on a pulmonary rehabilitation program, but who had declined to enroll or participate. All participants provided written consent prior to contributing to data collection for the study.

\section{Data collection}

Following kaupapa Māori, we looked to our participants to choose the preferred method for sharing of their views, experiences, and stories. ${ }^{38}$ For the participants recruited via the marae-based program, a focus group approach was chosen as the preferred method, with a single meeting involving all participants. This focus group was led by a Māori nurse researcher (BJ), who had an established working relationship with the health care provider delivering the pulmonary rehabilitation program, and who was known to people at the marae. The focus group was conducted following a regular pulmonary 
rehabilitation meeting at the marae, and included observance of tikanga Māori (Māori customs and protocols). For participants recruited via the mainstream, hospital-based programs, individual interviews were preferred. Two non-Māori researchers (PB and $\mathrm{MB}$ ) undertook these interviews at a location of each participant's choice. These interviews typically ran for 30-60 minutes and occurred either at the hospital or at the participant's home. Participants in individual interviews were invited to bring a support person along to the meeting if they wished. All data were collected between January 2012 and February 2013. The focus group ran for 90 minutes and was followed by informal conversation over a meal.

Semistructured questions were used as prompts for both the focus group and individual interviews. These were open ended but broadly centered on the following issues: how pulmonary rehabilitation was first introduced to the participants, how the participants made decisions to first attend pulmonary rehabilitation, factors that made attending and continuing with pulmonary rehabilitation easier or harder, what had happened to or for the participants as a consequence of attending pulmonary rehabilitation, and what pulmonary rehabilitation services should learn from the participants' experiences. Both the focus group and individual interviews were audio-recorded and transcribed verbatim. Self-identified ethnicity of each participant was collected using the 2006 New Zealand census question, handled in accordance with the Ministry of Health ethnicity protocols. ${ }^{44}$

\section{Data analysis}

Data from the interviews and focus group meeting were analyzed using constant comparative methods. ${ }^{35}$ NVivo software (QSR International, Melbourne, Australia) was used to help manage the interview data and data coding. Each transcript was read and reread, incorporating findings from additional data sources as the study progressed. Initial coding (open coding) was undertaken on a line-by-line basis, with subsequent analysis exploring the relationships between codes so as to develop higher-order concepts.

All initial interpretation of data was undertaken by two researchers (WL and BJ) before being discussed and peer reviewed by the whole research team as a group. Care was taken to ensure that interpretation of the data was undertaken from within a Māori worldview, with the interpretation provided by the Māori researchers (BJ and TI) directing the overall development of data analysis. To strengthen the credibility and trustworthiness of the analysis, and to ensure that the Māori community involved in the production of data maintained control over its interpretation, a meeting was held with participants and their community in March 2013 in which the preliminary analysis was presented and feedback was sought on the study findings. This meeting occurred on the marae and again incorporated observance of tikanga Māori. All Māori participants were invited to this meeting. Following this, the final write up of the study findings was completed.

In this paper, participant ID codes (such as F2, M11) have been included to distinguish between speakers without compromising anonymity.

\section{Results \\ Participant characteristics}

Twenty-five people with COPD participated in this study, including 13 men and 12 women aged between 40 and 79 years. All participants had received a clinical diagnosis of COPD, ie, clinically assessed for respiratory disease and found to have a forced expiratory volume in 1 second of less than $80 \%$ predicted by age and height. Although severity of COPD was not formally measured as part of the study, the participants had moderate to very severe COPD based on spirometric classification at time of referral to rehabilitation programs and were deemed eligible for pulmonary rehabilitation. ${ }^{45}$ Fifteen of the participants were Māori, eleven of whom were from the marae-based program, and three of whom were from the hospital-based program. One additional Māori participant had attended a hospital-based program previously (more than a year ago) but had declined to participate in a repeat pulmonary rehabilitation program when offered within the last 6 months. Of the eleven Māori participants from the marae-based program, four also had experience of attending a hospitalbased program.

Ten participants were identified as non-Māori and included nine New Zealand Europeans and one Samoan. Six of these ten people had participated in a hospital-based pulmonary rehabilitation program within the last 6 months. Three had attended a pulmonary rehabilitation class a year or more previously but had declined to attend a repeat pulmonary rehabilitation class when this had been offered in the last 6 months. Only one participant, a Samoan male, had never attended a pulmonary rehabilitation program, although he desired to do so (the primary reason given for not attending being that the timing of the program had conflicted with his vocational training program). We attempted to recruit additional people who had been offered a place on a pulmonary rehabilitation program yet had never attended and did not desire to do so, but these people $(n=18)$ did not take up invitations to participate in the study. 


\section{Overview of study findings}

Findings from this study demonstrated that while there were a number of factors influencing the uptake of pulmonary rehabilitation that were common to all participants, regardless of ethnicity or background, the experiences of the indigenous Māori participants differed from those of the non-Māori participants. Shared factors were grouped into four themes: 1) past experiences, 2) attitudes and expectations, 3) access issues, and 4) program experiences. For both hospital-based and marae-based programs, these factors could also be categorized in terms of those that influenced initial decisions to first attend pulmonary rehabilitation, those that influenced ongoing decisions to continue with a program once started, and those that did both. In addition, several factors were identified that moderated the participants' experiences and perspectives on these themes. These moderators included the involvement of family or whānau (extended family) or peers, interactions with health professionals, the approach to presenting information in brochures and invitation letters regarding specific programs, and the experience of new illness events.

For Māori participants, however, a number of unique perspectives and experiences also emerged from the data. Central to these was the importance of culturally meaningful connections. The most prominent of these connections was that of whanaungatanga, a Māori concept that can be broadly understood to mean relationships or kinship between people. Whanaungatanga also related to other cultural concepts that connected the people involved in delivering and participating in pulmonary rehabilitation, including: wairua (spirituality), whakapapa (genealogy), whānau (extended family), and kaupapa (the principles of the work of pulmonary rehabilitation). Within their pulmonary rehabilitation programs, many (but not all) Māori participants sought regular opportunities for making culturally meaningful connections between the people involved - a concept that can be loosely summarized by the term: whakawhanaungatanga (making relationships). When such opportunities were perceived as being absent in pulmonary rehabilitation programs, these Māori participants felt dissatisfied, were less inclined to join, or struggled to continue with these programs. An overview of the relationship between all of these factors and themes is presented in the Figure 1, with further discussion of these and extracts from the interview transcripts in the following sections. Additional extracts illustrating findings from this study are available in the Table S1.

\section{Common (cross-cultural) factors influencing uptake of pulmonary rehabilitation}

Past experiences of exercise, of health care services, and of life with COPD all had the capacity to influence the participants' decisions to attend pulmonary rehabilitation when it was first offered. Participants who had previously been fit and active earlier in their lives were often familiar with the gym setup of pulmonary rehabilitation programs and immediately felt comfortable with the idea of pushing themselves during exercise. Other participants had never enjoyed exercise, and, prior to turning up for the first time, were wary of how unpleasant they would find these programs.

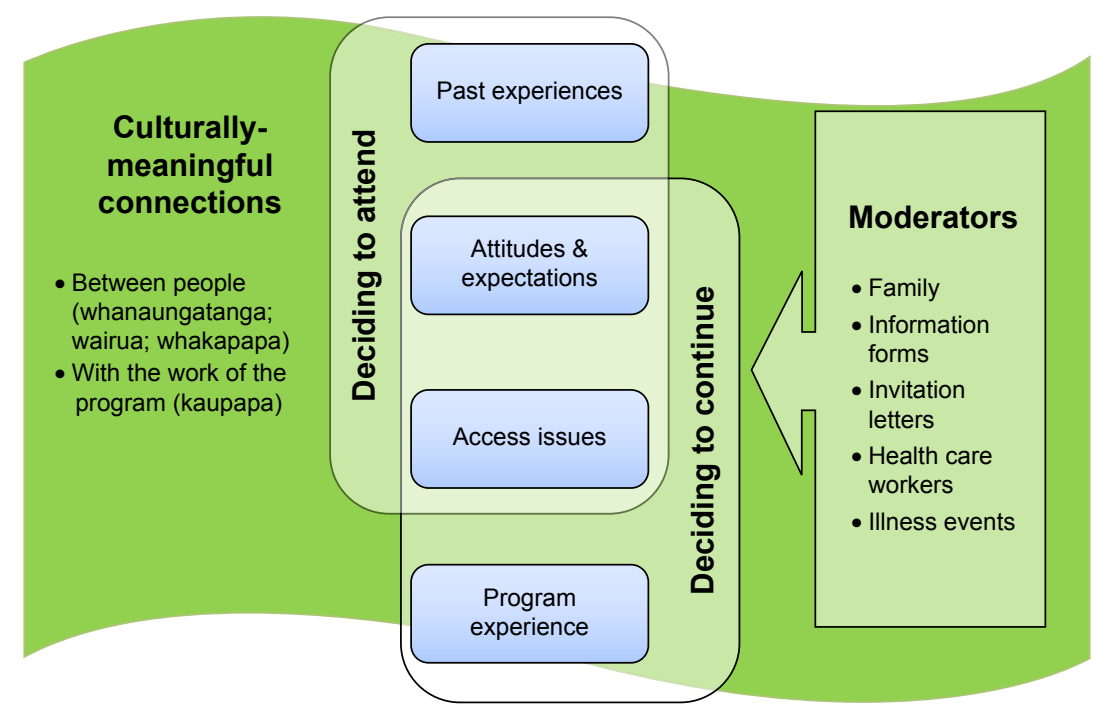

Figure I Factors contributing to decisions to attend and decision to stay in pulmonary rehabilitation program within a cultural context. 
Oh, that was the main thing that actually in a way almost put me off, "be active," I thought “oh, God.”... I'm not-I'm not a sports person... And no, I've never been particularly into exercising of any kind. I did go to a gym [once in the past]... I went but I can't honestly say I enjoyed it. [F2, female, NZ European, hospital-based program]

Past experiences of interactions with health professionals could also positively or negatively influence the participant's decision to attend pulmonary rehabilitation in the hospital setting. Māori participants in particular were inclined to recall negative interactions with non-Māori clinicians, making them less trusting of other health professionals who offer pulmonary rehabilitation within the hospital setting.

I had a hospitalization, went to see [the] doctor, and he told me basically I had less than five years to live. See you later. And that's how it was put to me... We still [had] a few questions for the surgeon, but it turned into "oh, it's a very busy clinic today." You know, I'm supposed to trust this man to open me up and take my lung out, and it's money, it all comes back to money... [M11, male, Māori, marae-based program]

Many participants also talked about the influence of their attitudes and expectations when it came to making decisions whether to begin or continue with pulmonary rehabilitation programs. Attitudes were often attributed to personal strengths or self-perceived flaws, eg, "I' $m$ the kind of person that once I make a commitment, I will do it". [F2, female, NZ European] versus “I'm just too proud to do stuff like that [catch the free shuttle to a hospital-based program]" [M23, male, Māori]. Expectations, both those facilitating uptake of pulmonary rehabilitation and those that were barriers, related to how the participants thought the program would be run and to how they thought they might respond physically and emotionally.

Access issues included the timing of programs, the duration of programs, issues around transport (particularly cost), and the distance to programs, as well as competing responsibilities. Participants ranged from those who were financially well off, were independent with transport, with no major additional responsibilities through to those who lacked personal transport, found public transport impossible, and had multiple competing responsibilities, including to work and family. Māori participants tended to have less capital resources than non-Māori participants, and so they tended to be more vulnerable to these practical matters. Indeed, one of the most successful strategies for increasing the uptake of both hospital-based and marae-based pulmonary rehabilitation programs by Māori appeared to be through the provision of transport.

I said to her "am I the only Māori coming?" And she said

"well there's meant to be others, but they're not coming."

And I said "probably because you have to find your own way." [F14, female, Māori, from the marae-based program, reflecting on time in a hospital-based program]

One hospital-based program provided free transport for Māori participants as part of a cultural support service run by the hospital, and this increased accessibility of the mainstream hospital-based program to Māori with COPD.

Program experiences naturally had a major impact on the participants' decisions to continue with pulmonary rehabilitation once started. These included initial experiences of positive health benefits arising from the programs, experiences of peer support and being part of a group, and interactions with the health professionals who delivered the programs.

\section{Cultural factors influencing uptake of pulmonary rehabilitation by Māori}

As already discussed, many of the Māori participants sought culturally meaningful connections with other people involved in the pulmonary rehabilitation programs that were offered, including those who had COPD and the program staff. While some Māori participants reported feeling welcomed and well accommodated by mainstream, hospital-based programs, a number of other Māori participants felt disinclined to attend programs where they might be the only Māori person attending.

How it was for me? - I felt very left out, I felt like being a Māori, you know. They were all in their own individual group, you know, the Pākehā [non-Māori New Zealanders]... I stuck out like a sore thumb, being the only Māori there. [F14, female, Māori, marae-based program, reflecting on hospital-based program]

In contrast, the marae-based program offered opportunities to participate in exercise and education within a context that served to both connect the Māori group members and to create a culturally familiar environment in which the participants could work on their health and well-being. Locating the program at a marae also inherently connected these participants to their wider community and to other health and social support services for which they may have been eligible and interested. Classes included tikanga (Māori protocol), 
waiata (singing) during exercise, and opportunities for kōrero (conversation) with peers who understood each other and who shared common cultural perspectives and life experiences.

And it just that whanaungatanga [connecting with others] time is very important, how everyone feels. It's something personal to yourself. But some get to say more than others... But it's a kind of down to our level, and it's good to bringing the tikanga aspect side of things, tikanga Māori, our waiata, how we do things, who with, and in a place that we feel good in being. [F12, female, Māori, marae-based program]

Considerable value was placed on the time that was spent after the formal class session on sharing stories with one another and with the program staff. This time centered on whakawhanaungatanga (ie, establishing and maintaining personal and culturally relevant connections) in a way that was more than just what might be considered casual conversation from a Western perspective. This included the use of a collective language, ie, te reo Māori (the Māori language), not being constrained by Western models of disease, validation of rongoa (traditional Māori medicine and healing methods), and having the autonomy to determine their own approach to mātauranga Māori (Māori knowledge).

The tikanga [protocol] aspect of it - we talk about our whakapapa [genealogy] first, that's all part of whanaungatanga [connection between people], and our stories are very important. Our stories are very important... When you come to the culture side of thing, you break that down even more to simple waiata, whakapapa, and te reo Māori, all that is our wellbeing. It's not just the having to learn what the Pākehā tell us to do. [F15, female, Māori, maraebased program]

Connection with things Māori was also considered highly important when first receiving information about pulmonary rehabilitation programs. This created an immediately positive first impression about the pulmonary rehabilitation program and a belief that it would be effective.

[Talking about the hospital brochure on pulmonary rehabilitation:] There's nothing that says "if you are Māori, this is what you need." It says you take part. And I was looking for the Māori side. And then I came here [the marae] and this was all Māori, and in the Māori environment, it - it was - for me, I thought oh, it must work. It has to. [M17, male, Māori, marae-based program]
In the hospital-based program, some aspect of whanaungatanga could be provided through the addition of cultural support services. For instance, while a free transport service was a practical way for some Māori to attend a mainstream, hospital-based program, it also provided an opportunity for Māori health professionals from the hospital to connect with Māori with COPD in the community. Time spent in a person's home when picking them up for transport and time spent in a hospital van driving between home and pulmonary rehabilitation became time for whakawhanaungatanga, rapport building, and information-sharing about personal health.

The lady [a Māori nurse] who picks us up [for pulmonary rehabilitation]... She asked me the question when she saw it [a non-invasive nasal ventilation machine for sleep apnea in the participant's home], and she said "do you use that?" And - and I looked at her. And she goes "no, you don't aye?" I went “no."... She happened to see it sitting there, and she asked me...

Interviewer: So you hadn't told anyone else you weren't using it?

No, I just kept quiet about it. I didn't know you had to come to the hospital after, and they check your machine, you see... So now I just felt relaxed with her. And yeah, I just - every question she asked I just answered it to my best knowledge. [F4, female, Māori, hospital-based program]

Another distinct characteristic of both the marae-based program and the support provided by cultural support services for people attending hospital-based programs was the time and energy spent by the Māori nurses on recruitment and retention of program attendees. The participants in this study describe the nurses associated with the marae-based program as being highly compassionate and caring but also particularly persistent when it came to encouraging involvement in the program. This time spent on connecting with participants, individually and culturally, assisted the health professionals overcome a heightened sense of initial suspicion held by some Māori participants regarding health services in general.

I didn't know these groups existed. I didn't even know [the marae] had a hauora [Māori health service]. Until she [the marae nurse] came to my place, or actually it was my partner they'd rung... and they came round. I'm looking - "Who are these two jungle bunnies come here?" And they told me - they read up on what I had you know. I said "Jesus, these people came straight off the street, they know what I got." So they ended up dragging me along - not dragging me, but getting me to come here, you know. [M17, male, Māori, marae-based program] 
The importance that the marae staff placed on the participants' program attendance made the participants feel valued, helped them see the potential benefits of the program, and contributed to their adherence to it. Although not all Māori participants required this type of intervention, a few attributed almost all of their current health and well-being to the efforts of the marae staff.

\begin{abstract}
All the Pākehā drugs, all the - the antibiotics, the prednisone - they were all messing with my weight, messing with my head, just put me in a dark place. And I didn't want to get out of bed. Didn't want to exercise. At that stage I just ready to give up. And then God sent me [the marae staff]. And it's those people that have got me to where I am now. [M16, male, Māori, marae-based program]
\end{abstract}

\section{Discussion}

To our knowledge, this is the first study that has explored cultural factors influencing the uptake of pulmonary rehabilitation. A number of barriers and facilitators of pulmonary rehabilitation were identified that are consistent with past research. ${ }^{14}$ These included the influence of travel and transport, attitudes and expectations (including beliefs regarding the potential benefits of pulmonary rehabilitation), and conflicts with other responsibilities - with these factors being moderated by the involvement of family members, health professionals, written information on programs, and ongoing illness events. ${ }^{14}$ However, for many (but not all) Māori participants, there were several additional factors that also influenced the successful uptake of pulmonary rehabilitation. In particular, Māori participants placed particularly high value on whakawhanaungatanga: the regular engagement in the making of culturally meaningful connections with others involved in the programs, and the ability to use a shared language. The use of health literacy approaches by health professionals resulted in an increased understanding of basic knowledge related to COPD and provided education on how to navigate the health system. This also played a role in the uptake and continuation of Māori in pulmonary rehabilitation programs.

Other researchers have previously theorized about the significance of social relationships when considering the mechanisms by which pulmonary rehabilitation achieves its positive effects. Halding et a ${ }^{46}$ explored the importance of social interactions in pulmonary rehabilitation programs in developing a sense of belonging for people with COPD, relating this to feelings of being better able to cope with the disease and to improved quality of life. The importance of social connection was also apparent in our data for both Māori and non-Māori people (Table S1), which we related to the influence of positive program experiences on uptake and continuation of pulmonary rehabilitation for people with COPD regardless of ethnicity. What our study adds to this notion is recognition of the need for such interactions to be culturally meaningful in order to be successful for the people involved. A distinction can be made between "social connections", which is a nonculturally specific term, and "whanaungatanga", which is a specific type of social connection that relates to customary Māori practices for the strengthening of kinship within groups, and which links these kinship ties to the cultural identity of Māori.

These findings have significant implications for the health sectors in New Zealand. Māori in New Zealand are admitted to hospital with COPD at a rate of 4.03 per 1,000 people annually, and have an age-standardized admission rate for COPD that is 4.4 times higher than for non-Māori. ${ }^{47}$ Pulmonary rehabilitation is an immensely important intervention for all people with COPD, ${ }^{12}$ but particularly for Māori given their higher rates of disease burden. It is therefore fundamentally important that respiratory services are responsive to the needs of Māori with COPD. This includes minimizing any barriers to Māori attending pulmonary rehabilitation, including cultural barriers. Our study suggests that spending time on culturally meaningful communication and relationship building is a key part of this process. By not offering culturally safe pulmonary rehabilitation, health services may fail to address the needs of a group of people with COPD who could significantly benefit from those services.

Teachings on cultural safety highlight the importance of examining the culture of the organizations that provide health care services, and the experience of these by people from minority groups..$^{29,31,33,34}$ There can be a tendency for dominant cultures in any country to assume that their mainstream health care services are somehow acultural - a kind of tabula rasa (ie, a neutral platform) for the fair and equitable provision of treatments and interventions for all people that they serve. The findings from this study demonstrate that hospital-based pulmonary rehabilitation services can feel unwelcoming or unsafe for some Māori members of the COPD community in New Zealand. It seems likely that similar issues may exist for indigenous, minority groups in other countries, although further studies would be required to test this assumption. These issues could be addressed by mainstream health providers examining and addressing problems experienced by nonmainstream cultural groups, or by the provision of services specifically tailored for these 
groups. Our study, for instance, highlights the potential value of programs based on Māori models of health that deliver culturally appropriate services to indigenous people with COPD in New Zealand. The marae-based service examined in this study provides one example of a "by Māori, for Māori" program that has improved opportunities for Māori with COPD to benefit from pulmonary rehabilitation. It is also important, however, to recognize the benefits and responsibility of mainstream health care providers to also endeavor to provide culturally responsive services. One relatively simple example of this from our study is the value of facilitating pulmonary rehabilitation attendance by Māori through the provision of free transport.

In terms of study limitations, we acknowledge that the involvement of non-Māori researchers in kaupapa Māori studies is considered controversial by some. ${ }^{36,48}$ However, we have followed the thinking and recommendations of Māori researcher, Russell Bishop, who argued that non-Māori involvement in Māori-controlled research within Māori space, when undertaken appropriately, can be considered both koha (a gift) for Māori communities and an obligation under Te Tiriti o Waitangi (the Treaty between the British Crown and Māori, signed in 1840, which still guides political and public activity in New Zealand today). Reflexivity by the researchers, both Māori and non-Māori, included feedback directly from the Māori participants and Māori health care providers, and was used to ensure that the research stayed consistent with kaupapa Māori.

As a qualitative study, the local influences on this study (eg, the New Zealand health care system, local attitudes and commitment to indigenous peoples' health, etc) need to be considered when evaluating the transferability of our findings. Also of note, missing from our data are the experiences of people who declined to take up any opportunity to participate in pulmonary rehabilitation when these were offered. Nevertheless, it is likely that the central ideas driving this research will resonate with respiratory services aiming to improve the health of indigenous peoples internationally. An additional limitation of this study is that non-Māori participants were not offered the opportunity to comment on the preliminary analysis of their study data. This means that our analysis of non-Māori perspectives does not include this check of trustworthiness.

Research on indigenous health care is sorely lacking. It has been argued in other countries that the lack of research on issues to do with indigenous health suggests that the degree of political will and attention to such matters are incommensurate with the seriousness of the problem. ${ }^{49}$ Furthermore, a recent systematic review concluded that there is urgent need for cost-effectiveness studies to be conducted on indigenous health care interventions in order to better understand their value and to further develop these models of care. ${ }^{50}$ With COPD being the fifth leading cause of total years with disability worldwide, ${ }^{51}$ this is particularly true of interventions for indigenous peoples with COPD.

\section{Conclusion}

Pulmonary rehabilitation is a highly successful intervention for people with COPD. However, pulmonary rehabilitation is only effective if programs are designed and implemented in a manner that encourages participants to engage regularly with them. Past research has highlighted a number of factors influencing the uptake and completion of pulmonary rehabilitation, and many of these appear to be relevant regardless of the culture or country of the people participating in these programs. In this study, we have demonstrated that in addition to these factors, cultural issues specific to indigenous community groups can also have a key influence over the way pulmonary rehabilitation is experienced and responded to. Failure to include cultural practices in the delivery of mainstream pulmonary rehabilitation may act as a barrier to the uptake and participation of indigenous, minority peoples. This research also highlights the potentially important value of indigenous-led pulmonary rehabilitation programs for indigenous, minority groups. Further research on culture, ethnicity, and the management of COPD is urgently required.

\section{Acknowledgments}

This study was funded by the Asthma Foundation, New Zealand. We thank all the participants and staff who contributed to this study from Tu Kotahi Māori Asthma Trust, Capital \& Coast District Health Board, and Hutt Valley District Health Board.

\section{Disclosure}

The authors report no conflicts of interest in this work.

\section{References}

1. World Health Organization. Indigenous Populations. Geneva, Switzerland: World Health Organization; 2014. Available from: http://www.who.int/ topics/health_services_indigenous/en/. Accessed February 10, 2016.

2. Gracey M, King M. Indigenous health part 1: determinants and disease patterns. Lancet. 2009;374:65-75.

3. Anderson I, Crengle S, Leialoha Kamaka M, Chen TH, Palafox N, Jackson-Pulver L. Indigenous health in Australia, New Zealand, and the Pacific. Lancet. 2006;367(9524):1775-1785.

4. Bjerregaard P, Young TK, Dewailly E, Ebbesson SO. Indigenous health in the Arctic: an overview of the circumpolar Inuit population. Scand J Public Health. 2004;32(5):390-395. 
5. Bramley D, Hebert $\mathrm{P}$, Tuzzio L, Chassin M. Disparities in indigenous health: a cross-country comparison between New Zealand and the United States. Am J Public Health. 2005;95(5):844.

6. Vos T, Barker B, Begg S, Stanley L, Lopez AD. Burden of disease and injury in Aboriginal and Torres Strait Islander Peoples: the indigenous health gap. Int J Epidemiol. 2009;38(2):470-477.

7. Shirtcliffe P, Weatherall M, Marsh S, et al. COPD prevalence in a random population survey: a matter of definition. Eur Respir J. 2007; 30:232-239.

8. Town I, Taylor R, Garrett J, Patterson J, editors. The Burden of COPD in New Zealand. Wellington, New Zealand: Asthma and Respiratory Foundation of New Zealand Inc.; 2003.

9. Telfar L, Baker M, Pierse N, Zhang J. The Impact of Respiratory Disease in New Zealand: 2014 Update. Wellington, New Zealand: The Asthma Foundation; 2015

10. Levack W, Weatherall M, Reeve J, Mans C, Mauro A. Uptake of pulmonary rehabilitation in New Zealand by people with chronic obstructive pulmonary disease in 2009. $N Z$ Med J. 2012;125(1348):1-11.

11. Desveaux L, Janaudis-Ferreira T, Goldstein R, Brooks D. An international comparison of pulmonary rehabilitation: a systematic review. COPD. 2015;12(2):144-153.

12. McCarthy B, Casey D, Devane D, Murphy K, Murphy E, Lacasse Y. Pulmonary rehabilitation for chronic obstructive pulmonary disease. Cochrane Database Syst Rev. 2015;2:CD003793.

13. Puhan MA, Gimeno-Santos E, Scharplatz M, Troosters T, Walters E, Steurer J. Pulmonary rehabilitation following exacerbations of chronic obstructive pulmonary diesease. Cochrane Database Syst Rev. 2011; 10:CD005305.

14. Keating A, Lee A, Holland AE. What prevents people with chronic obstructive pulmonary disease from attending pulmonary rehabilitation? A systematic review. Chron Respir Dis. 2011;8(2):89-99.

15. Nici L, Donner C, Wouters E, et al. American thoracic society/European respiratory society statement on pulmonary rehabilitation. Am J Respir Crit Care Med. 2006;173(12):1390-1413.

16. Reid P, Robson B, Jones CP. Disparities in health: common myths and uncommon truths. Pac Health Dialog. 2000;7(1):38-47.

17. Statistics New Zealand. New Zealand Period Life Table: 2012-14; 2015. Available from: http://www.stats.govt.nz/browse_for_stats/ health/life_expectancy/NZLifeTables_HOTP12-14/Commentary. aspx\#narrowing-gap-maori. Accessed December 17, 2015.

18. King M, Smith A, Gracey M. Indigenous health part 2: the underlying causes of the health gap. Lancet. 2009;374(9683):76-85.

19. Harris R, Cormack D, Tobias M, et al. The pervasive effects of racism: experiences of racial discrimination in New Zealand over time and associations with multiple health domains. Soc Sci Med. 2012;74(3): $408-415$.

20. Marrone S. Understanding barriers to health care: a review of disparities in health care services among indigenous populations. Int J Circumpolar Health. 2007;66(3):188-198.

21. Jones CP. Levels of racism: a theoretic framework and a gardener's tale. Am J Public Health. 2000;90(8):1212.

22. Krieger N. Does racism harm health? Did child abuse exist before 1962 ? On explicit questions, critical science, and current controversies: an ecosocial perspective. Am J Public Health. 2003;93(2):194-199.

23. McDonald SP. End-stage kidney disease among indigenous peoples of Australia and New Zealand. Kidney Int Suppl. 2013;3(2):170-173.

24. Gao S, Manns BJ, Culleton BF, et al. Access to health care among status Aboriginal people with chronic kidney disease. CMAJ. 2008;179(10): 1007-1012

25. Castor ML, Smyser MS, Taualii MM, Park AN, Lawson SA, Forquera RA. A nationwide population-based study identifying health disparities between American Indians/Alaska Natives and the general populations living in select urban counties. Am J Public Health. 2006;96(8):1478-1484.

26. Heaman MI, Gupton AL, Moffatt ME. Prevalence and predictors of inadequate prenatal care: a comparison of aboriginal and nonaboriginal women in Manitoba. J Obstet Gynaecol Can. 2005;27(3): $237-246$.
27. Shah BR, Gunraj N, Hux JE. Markers of access to and quality of primary care for aboriginal people in Ontario, Canada. Am J Public Health. 2003; 93(5):798-802

28. Signal V, SarfatiD, Cunningham R, Gurney J,Koea J,Ellison-LoschmannL. Indigenous inequities in the presentation and management of stomach cancer in New Zealand: a country with universal health care coverage. Gastric Cancer. 2015;18(3):571-579.

29. Papps E, Ramsden I. Cultural safety in nursing: the New Zealand experience. Int J Qual Health Care. 1996;8(5):491-497.

30. Wepa D. Cultural Safety in Aotearoa New Zealand. 2nd ed. Melbourne, Victoria: Cambridge University Press; 2015.

31. Jungersen K. Cultural safety: Kawa Whakaruruhau - an occupational therapy perspective. N Z J Occup Ther. 2002;49(1):4-9.

32. Leininger MM. Transcultural Nursing: Concepts, Theories, and Practices. New York, NY: Wiley; 1978.

33. Ramsden I. Cultural safety/Kawa Whakaruruhau ten years on: a personal overview. Nurs Prax N Z. 2000;15(1):4-12.

34. Richardson S. Aotearoa/New Zealand nursing: from eugenics to cultural safety. Nurs Inq. 2004;11(1):35-42

35. Charmaz K. Constructing Grounded Theory. London, UK: Sage Publications Ltd; 2006.

36. Walker S, Eketone A, Gibbs A. An exploration of kaupapa Maori research, its principles, processes and applications. Int $J$ Soc Res Methodol. 2006;9(4):331-344.

37. Henry E, Pene H. Kaupapa Maori: locating indigenous ontology, epistemology and methodology in the academy. Organization. 2001;8(2): 234-242.

38. Jones B, Ingham TR, Cram F, Dean S, Davies C. An indigenous approach to explore health-related experiences among Māori parents: the Pukapuka Hauora asthma study. BMC Public Health. 2013;13(1):228.

39. Smith LT. Decolonizing Methodologies: Research and Indigenous Peoples. London, UK: Zed Books; 1999.

40. Jones B, Ingham T, Davies C, Cram F. Whānau Tuatahi: Māori community partnership research using a Kaupapa Māori methodology. Mai Rev. 2010;3(1):1-14.

41. Willig C. Introducing Qualitative Research in Psychology. 3rd ed. Berkshire, London: Open University Press; 2013.

42. Crotty M. The Foundations of Social Research: Meaning and Perspective in the Research Process. New South Wales, Australia: Allen \& Unwin; 1998.

43. Hudson M, Milne M, Reynolds P, Russell K, Smith B. Te Ara TikaGuidelines for Maori Research Ethics: A Framework for Researchers and Ethics Committee Members. Auckland, New Zealand: Health Research Council of New Zealand; n.d.

44. Ministry of Health. Ethnicity Data Protocols for the Health and Disability Sector. Wellington, New Zealand: Ministry of Health; 2004.

45. Global Initiative for Chronic Lung Disease (GOLD). Global strategy for the diagnosis, management, and prevention of chronic obstructive pulmonary disease. Medical Communication Resources, Inc.; 2015. Available from: http://www.goldcopd.com/. Accessed December 17, 2015.

46. Halding A, Wahl A, Heggdal K. 'Belonging'. Patients' experiences of social relationships during pulmonary rehabilitation. Disabil Rehabil. 2010;32(15):1272-1280.

47. Milne RJ, Beasley R. Hospital admissions for chronic obstructive pulmonary disease in New Zealand. N Z Med J. 2015;128:23-35.

48. Bishop R. Whakawhanaungatanga: Collaborative Research Stories. Palmerston North, New Zealand: Dunmore Press Ltd; 1996.

49. Derrick GE, Hayen A, Chapman S, Haynes AS, Webster BM, Anderson I. A bibliometric analysis of research on Indigenous health in Australia, 1972-2008. Aust N Z J Public Health. 2012;36(3):269-273.

50. Angell BJ, Muhunthan J, Irving M, Eades S, Jan S. Global systematic review of the cost-effectiveness of indigenous health interventions. PLoS One. 2014;9(11):e111249.

51. Vos T, Flaxman AD, Naghavi M, et al. Years lived with disability (YLDs) for 1160 sequelae of 289 diseases and injuries 1990-2010: a systematic analysis for the Global Burden of Disease Study 2010. Lancet. 2013;380(9859):2163-2196. 


\section{Supplementary material}

Table SI Extracts from transcripts illustrating culturally shared factors influencing decisions to start or continue with pulmonary rehabilitation

\begin{tabular}{lll}
\hline Influencing factors & Facilitating uptake of pulmonary rehabilitation & $\begin{array}{l}\text { Barriers to uptake of pulmonary } \\
\text { rehabilitation }\end{array}$ \\
\hline
\end{tabular}

\section{Past experiences}

Of exercise

Of health services

Attitudes and expectations continued...

Of having COPD
The course it's really like a circuit training thing, which I could relate to that, because I have been absolutely super fit in my time... I'm actually familiar with circuit training. Lots and lots of circuit training. [M3, male, NZ European, hospitalbased program]

Oh, I had um - on one of our courses to do with my diabetes... and I ended up with um, being wheel-chaired, ah, to A\&E [Accident \& Emergency]. Cause I had a - my muscles pulled and that, I couldn't - [it] was like - sciatica. So, went to the doctors, the only thing they said is um, tramadol... [So for pulmonary rehabilitation in the hospital] You're in the right place. If anything goes down. [F5, female, Māori, hospital-based program]

\section{Access issues}

Timing of program
It came to a head 3 years ago when I was on a flight from Auckland to Vancouver. And the last three hours of that flight - it's a I4-hour flight - I couldn't breathe. I just spent three hours in the bathroom with my Ventolin inhaler. And that really scared me... And I was in hospital for 4 days when I arrived. [MI, male, NZ European, talking about the event that triggered deciding to eventually attend hospital-based program]

Nobody else can help me but myself, so I thought it was time to kick myself in the butt, because okay, I weighed in at a hundred and twenty-six (kilograms). [F4, female, Māori, hospital-based program]

I thought, you know, if this is going to be helpful to me, and restore some of my quality of life, then it's worth doing. [MI, male, NZ European, hospital-based program]

There's plenty of time to catch the buses [to get to pulmonary rehabilitation]. Cause it - it didn't start till half past ten o'clock. [M6, male, Māori, hospital-based program]
Was there anything about the class that made you nervous before you started, or when you first started going?

The exercise. I used to think "oh," you know. And the physio will say "no, keep going, keep going," you know, you think "I can't breathe." I used to be afraid of the breathing. [F5, female, Māori, hospital-based program]

I didn't like it... They said I had to do this; do that. But they didn't explain anything about the health, what it does to you...

What stopped you coming back?

I went to ah, that department, ah, where you blow these things in the tube... I chucked it in too, that one. Pissed me off... Well they told me to blow, blow, blow, blow, keep blowing. And that was - I had a severe - ah, breathing problems! So I chucked it in. [M6, male, Māori, hospital-based program] And that exercise in the hospital gym was rubbish. We had to be there at nine o'clock in the morning, I mean anyone with COPD or severe emphysema know, that's not a really for us. It takes me over an hour just to have a shower. [MI6, Māori, maraebased program]

I used to have a bad attitude. I used to think, oh, exercise ain't going to help me, you know... At first I really had a bit of a negative attitude to it. Cause I have negative attitude to everything. And I'm like “nah, I don't want to go to that." You know, I lose interest in things quick. Real quick. [F5, female, Māori, hospital-based program]

I mean that's why I wasn't there at the end of last year, cause I was still in that dark place, and I was finding excuses every week, why l'm not coming. Even though [the community nurse] and them are trying to push me here, and push me here. Because I wasn't in the right head space for myself, I didn't want to bring anyone else down. [MI6, male, Māori, marae-based program]

I found that early morning was not good for me. I couldn't concentrate on what they were doing. And the last thing I wanted to do was walk. [MI I, male, Māori, marae-based program, reflecting on time in hospital-based program]

They sort of said to me well if I wanted to, I could go for this course, and there was this one and that one, and I sort of thought: well I don't want to be doing it in the middle of winter. [F2, female, NZ European, hospital-based program]

(Continued) 
Table SI (Continued)

\begin{tabular}{ll}
\hline Influencing factors & Facilitating uptake of pulmonary rehabilitation \\
\hline Duration of program & If it was I 2 weeks l'd still come. If it was 6 weeks l'd still \\
come, you know. [MI, male, NZ European, hospital-based \\
program] \\
Attending the class all the time, and it's only an 8 weeks \\
course, it's not long enough for me though. I love it. [F4, \\
female, Māori, hospital-based program]
\end{tabular}

Transport \& distance to program

Competing responsibilities

\section{Program experiences} Health effects

Being part of a group

Peer support

Health professional support
Do you think you would be coming if it wasn't for the [transport service] support?

Nah, I wouldn't have been coming... Cause I can't get around - well I can, but I don't want to. You know, they pick me up and drop me off. [F5, female, Māori, hospital-based program]

It's also very handy for me, I don't live far away. And I have the car, and I have a disability parking space, that's handy. And I can park in the disability parking space and walk the rest of the way. So it's convenient for me to come to the hospital. [F8, female, NZ European, hospital-based program]

I'm a contractor, so I get paid by the hour. So I just had to say to my boss over the next 8 weeks l'll be away... these Mondays and Thursdays, away from the office [MI, male, NZ European, hospital-based program]

[To start with] I just thought "oh, what we're going - going to get out of it?" But I thought l'll keep going. But then I said to them, when l'd been to it for a few times, I used to say, "oh, I must admit, it has been doing me good." I said - before I used to think oh exercise was just a load of - it's not going to help you, you know, with all the breathlessness and all that. It's not going to help you. [F5, female, Māori, hospitalbased program]

I like the atmosphere... And although we do exercise, and oh, we all hate doing them. But we do, we put an effort into them... And I love it up here, I love coming here, it's just the atmosphere's totally different [from hospital], and it's beautiful. [FI4, female, Māori, marae-based program] There was a group of us that um, we had so much fun on [the pulmonary rehabilitation program]... we've stuck together as a group. And um, and we go out for coffee and we have lunches and, and ah, we go to [the community gym] too once a week. [F22, female, NZ European, hospital-based program]

Once you're in this class, and you hear the korero (Māori word for "conversation") around the table, you do, you get over what you're feeling, because there's others behind you that are feeling either worse, or better. And its just - for me, it was sort of like a - a healing part for me. [FI4, female, Māori, marae-based program]

They [the health professionals] were the ones to encourage you on, and to - they actually showed you how, and if there was something you couldn't do... they found something else... And then they'd make sure that: "no, you need to stand with your feet a bit further apart," or "lean into it more," or whatever. Whereas when you in a gym you don't get any of that. [F2, female, NZ European, hospital-based program]
Barriers to uptake of pulmonary rehabilitation

And it was a 9 week course. That was the other thing that put me off a little bit. Nine weeks. I sort of started off by sort of thinking, "oh, it's only 9 weeks." And then I sort of started realizing how long 9 weeks was... Well hang on a minute; I could be doing other things. [F2, female, NZ European, hospital-based program] I said to her "am I the only Māori coming?" And she said "well there's meant to be others, but they're not coming." And I said "probably because you have to find your own way [FI4, female, Māori, from the marae-based program, reflecting on time in the hospital-based program.]

I mean I know that going there might help but sometimes when you get out, when you can't get out of bed or you can't shower, it's just l'm stuck to the oxygen. I have to stay home. I can't do anything, that's the only reason why I wouldn't go. [M23, male, Māori, hospital-based program, decline recent opportunity to attend]

Because I work, I had to actually change days that I worked and everything to be able to attend it... There were a couple of times even then that, because of work, I couldn't go. [F2, female, NZ European, hospital-based program]

Yeah, and my last class I couldn't do their stair exercise, and it's only four steps. Yeah, and that, that, that disheartens me as well. You know, I can't even do four steps, and then you watch um, the elderly lady going up. It makes me angry that l've got myself in that position. Yeah. But yeah, I know that that's my only way out, is to actually go to those classes. [M23, male, Māori, hospital-based program] Basically, the biggest thing I struggled with was that I was. I'm only 42. And how many people 40, 60, 70, 80 year olds [were at pulmonary rehabilitation]? I struggled. [MI6, male, Māori, marae-based program] I think there's always a certain amount of embarrassment to - to show yourself, and what you can't do, often, in front of other people... So there was a certain sense of reserve about it. [F8, female, NZ European, hospital-based program]

I went to like 3 or 4 classes in a row, which is like 3 or 4 weeks. But then I started getting a little bit um, probably a bit too proud, or thinking about nah these, these people are too old for me, or the other way around, I'm too young. [M23, male, Māori, hospital-based program] 
International Journal of COPD

\section{Publish your work in this journal}

The International Journal of COPD is an international, peer-reviewed journal of therapeutics and pharmacology focusing on concise rapid reporting of clinical studies and reviews in COPD. Special focus is given to the pathophysiological processes underlying the disease, intervention programs, patient focused education, and self management protocols.

This journal is indexed on PubMed Central, MedLine and CAS. The manuscript management system is completely online and includes a very quick and fair peer-review system, which is all easy to use. Visit http://www.dovepress.com/testimonials.php to read real quotes from published authors 